\title{
Motivations of participants in the citizen science of microbiomics: data from the British Gut Project
}

\author{
Lorenzo Del Savio', Barbara Prainsack² and Alena Buyx ${ }^{1}$
}

Purpose: The establishment of databases for research in human microbiomics is dependent on the recruitment of sufficient numbers and diversity of participants. Factors that support or impede participant recruitment in studies of this type have not yet been studied.

Methods: We report the results of a survey aimed at establishing the motivations of participants in the British Gut Project, a research project that relies on volunteers to provide samples and to help fund the project.

Results: The two most frequently reported motivations for participation were altruism and solidarity. Low education levels appeared to be a recruitment obstacle. More than half of our 151 respondents said they would participate in further citizen-science projects; $38 \%$ said they would not participate in a similar project if it was for-profit or in a project that did not release data sets in repositories accessible to scientists (30\%).

Conclusions: The desire to take part in research was reported as a key motivation for participation in the British Gut Project (BGP). Such prosocial motivations can be mobilized for the establishment of large data sets for research.

Genet Med advance online publication 26 January 2017
The establishment of databases for research in human microbiomics poses important recruitment challenges. The interindividual diversity of microbiota composition is vast, and the microbiome is a dynamic research object that changes in response to environmental, physiological, and pathological events. ${ }^{1-3}$ Microbiomic studies require large cohorts, repeated sampling from the same individuals, rich phenotypic data, and dynamic behavioral or clinical annotation.,

The American Gut Project and its British offshoot, the British Gut Project (BGP), have used a "citizen-science" approach to establishing a database on human microbiomics. ${ }^{6}$ Citizen science is a broad label under which a wide range of practices are subsumed, ranging from volunteers contributing money or collecting data to volunteers running an entire project without the involvement of professional scientists. In the particular instance of the American Gut Project and the BGP, the projects use a "thin" model of citizen science whereby the project's goals and methods are determined by professional scientists. Volunteers are enlisted to contribute samples and funding. Although there are other, more "robust" practices and understandings of citizen involvement in science, ${ }^{7}$ the BGP is similar to citizen-science projects in the natural sciences, whereby lay people contribute to the collection and annotation of data (e.g., environmental data and species observations).

The gut projects use FundRazr, a dedicated crowdfunding platform. In fact, the American Gut Project and the BGP are among the platform's most successful campaigns, recently hitting the $\$ 1$ million threshold. Upon subscription to the platform and payment, volunteers receive a swabbing kit that they must return by mail. Within a few weeks, they receive their test results online. Standard analysis includes information on microbial species found in a person's gut and how this person's gut microbes compare with those of other individuals. The project emphasizes that test takers should not take their personal results as clinical advice.

Data are de-identified and released through the European Bioinformatics Institute, a member of the International Nucleotide Sequence Database Consortium. ${ }^{8}$ Test takers can access their own raw data using a unique identifier code.

The BGP combines features of direct-to-consumer (DTC) genomic testing (i.e., the sale of tests directly to paying consumers without the involvement of clinicians) and citizen science in the sense that "lay" people participate in research tasks. Unlike with DTC genomics, the disclosure of personalized test results to users is not the main sales pitch in the case of the gut projects. The main argument in the BGP's recruitment strategy is that users can contribute to science. This raises the question of whether this model is suitable to support recruitment in other research projects in the biomedical field.

To answer this question, we need to understand why participants decide to take part and their views of the BGP's aims and organization. To our knowledge, a study of these aspects in microbiomics has not been carried out so far (similar studies have been conducted for DTC genomics ${ }^{9,10}$ ). An ex-post survey of motivations is not a perfect way to understand why people decided to take part. It is, however, an effective way to identify

The last two authors contributed equally to this work.

${ }^{1}$ Division of Biomedical Ethics, Institute of Experimental Medicine, University of Kiel, Kiel, Germany; ${ }^{2}$ Global Health \& Social Medicine, King’s College London, London, UK. Correspondence: Alena Buyx (a.buyx@iem.uni-kiel.de) 
important themes and values that matter to participants in connection with their participation.

\section{MATERIALS AND METHODS}

In December 2015, after obtaining research ethics approval, we contacted 370 participants in the BGP who had agreed to be recontacted through a dedicated BGP mailing list. Between December 2015 and January 2016, 151 participants completed an online questionnaire that was circulated via e-mail and hosted by the survey service SurveyMonkey. The survey consisted of 10 closed questions. The participation rate was $41 \%$; the demographic characteristics of the responders are reported in Supplementary Figure S1 online. We also obtained the aggregate demographic data of all BGP participants, which enabled us to compare the characteristics of the entire set of BGP participants with those who participated in our survey.

\section{RESULTS}

The two most common motivations for participation in the BGP were prosocial: to participate in clinically useful research (78\%, $n=116)$ and to help clinicians in the search of cures for medical conditions $(79 \%, n=118)$. The desire to improve self knowledge and lifestyle figured prominently but to a lesser extent: $39 \%(n=56)$ declared they wanted to find a cure for their condition, and 58\% $(n=87)$ and $59 \%(n=88)$, respectively, said they wanted to learn how to change their diet or find out whether they are healthy. Less than $20 \%(n=29)$ declared that they changed their diet after obtaining results or consulted a medical doctor. Supplementary Figure S2 online summarizes these findings.

$74(53 \%)$ respondents said that they plan to participate in another citizen-science project, and $40 \%(n=57)$ said they have not yet decided whether to do so; $37(n=43)$ and $34 \%(n=53)$, respectively, said they would not participate in a for-profit project or a project that does not release data sets in public repositories. 116 respondents (82\%) said that the academic nature of the BGP played a role in their decision to participate. 99 said they believed that there are no risks in projects such as the BGP $(71 \%)$. Only $1 \%(n=2)$ said the risk is very high.

Some respondents had not received results at the time of our survey. They declared this in the comments section and did not answer questions pertaining to the use of results. Approximately 1 in 10 said they consulted a physician as a result of undergoing the test $(11 \%, n=15)$, and 2 in $10(21 \%, n=29)$ declared they changed their dietary habits. One-quarter of all respondents $(25 \%, n=35)$ said they understood their condition better after taking the test. Almost 6 in $10(57 \%, n=81)$ said they believed that their knowledge of microbiomics was sufficient to interpret results correctly.

\section{DISCUSSION}

The findings from our research need to be extrapolated with caution to the broader set of participants in the BGP. Most of the respondents to our survey were 65 years old or older, but participants in the BGP are more evenly distributed across age groups. Moreover, although the participation rate was high in relation to comparable studies, ${ }^{10}$ our sample size was small and no significant associations could be found between demographic variables and answers to specific questions. It is also important to remember that participants in the survey represent a subset of only the participants in the BGP who chose to be recontacted. The motivations and values of these participants might differ from those of the other participants in the BGP because willingness to be recontacted could be a proxy measure of a positive attitude toward being part of a citizenscience project.

Participants in our study reported that prosocial motivations played an important role in their willingness to participate in the BGP. Such motives are usually described as altruistic or solidaristic. Altruism is typically defined as a helping behavior based on a broad interest to further common, prosocial goals or as prioritizing the needs of others over one's own. Solidarity can be defined as a shared commitment to accept "costs" (e.g., financial, practical, or emotional) to support others to whom people recognize relevant similarities, such as a shared interest in research or a shared health risk or experience with illness. ${ }^{11}$ In other words, although altruism describes a person's disposition toward others, solidarity is a practice that draws on and reinforces connections between people.

Prosocial motivations have been shown to underlie participation in biomedical research, ${ }^{12}$ although scholarship on this topic has been criticized as overemphasizing altruistic behavior. ${ }^{13}$ Against this backdrop, the notion of solidarity seems to better accommodate the simultaneity of self-interest and concern for others that has been found to motivate research participation in many studies. ${ }^{14}$ In particular, in the context of data-rich medicine, solidarity has been described as an important motive that could be harnessed to enjoin people to participate in such research as well as a powerful way to frame such research endeavors. ${ }^{15}$

Also in our study, both self-interest and prosocial motivations were at play. In our case, however, direct benefits or clinical relevance of the research seem to have played a minor role; although self-knowledge was a motivation for some participants, only a minority would have refrained from taking part if the tests had had no personal health relevance. We can only speculate about the reasons for this. It could be that the strong citizen-science rhetoric in the advertisement of the BGP, which calls on potential participants to "help science" at the same time that they "explore their inner self" (http://britishgut.org), is particularly attractive to people looking for an opportunity to support science as an added benefit to getting personalized results. More than one-third of our respondents said that they would not participate in a similar project within a commercial setting or where data would not be released for public benefit, which indicates that public benefit was indeed considered an important factor. A recent study on public attitudes toward commercial data use in the United Kingdom yielded similar findings. ${ }^{16}$ It is therefore possible that for projects like the BGP to thrive, an emphasis on public benefits can help with recruitment.

Another finding of our study that is relevant to the design of similar studies is that most respondents considered risks 
associated with participation in the BGP to be nonexistent or low (see Supplementary Figure S2 online). This is an important indication that volunteers in projects such as the BGP or consumers of DTC genomics, who have been shown to be highly educated individuals, ${ }^{17}$ do not expect to be harmed by their participation in CS projects of this kind. Apparently, participants themselves are-at least in our study-far less concerned about the risks of such projects than some outside commentators. ${ }^{18}$ Overall, because direct benefits (clinical relevance) did not play any decisive role in motivating participation, the type of project we studied appears to have a very positive risk-to-benefit ratio in the eyes of participants. Only 13 of 151 respondents mentioned risk concerns. Only 7 respondents described specific risks, including data leakage, failure of anonymization, and bioterrorism, that they did not deem significant enough to deter them from participating in the BGP.

We found that age and education level seemed to be important factors in recruitment, with poorer recruitment among the young and less educated $(7 / 10(n=99)$ respondents to our survey hold at least a college-or comparable-degree, whereas the overall figure for the United Kingdom is closer to 3/10). Given that bioresources reliant on self-recruitment of participants are already biased toward the resource-rich, ${ }^{19}$ this bias may be exacerbated, especially in light of the accompanying crowdfunding campaigns and the financial costs that participants shoulder in these studies.

Four drivers of the well-known decline in research participation have been identified in the literature: an increase in the numbers of studies conducted, public mistrust of science, the burden of the studies for participants, and a general decrease in volunteerism. ${ }^{20}$ Citizen-science approaches can help address the last three factors. Participatory designs can improve scientific literacy and give people more control over the aims of the study, thereby helping to address lack of trust. The burdensome nature of some studies might be countered by the desire for participation. Finally, in addition to utilizing new forms of digital interaction, our data indicate that decreasing volunteerism could be addressed by emphasizing prosocial reasons for participation in the recruitment strategies of citizen-science projects such as the BGP.

The desire of people for active participation in research can and should be mobilized and expanded. To ensure that marginalized groups in society are represented in studies that are likely to lead to health-related inferences at the population level, this should be accompanied by recruitment efforts tailored specifically to underserved groups, although different incentives may be required. In the case of crowdfunding, several participation formats should be offered, preferably encompassing "entrylevel" packages at the lowest possible cost. Overall, engaging participants more directly in funding, designing, and evaluating projects as well as in collecting samples and data could help ensure the continuation and expansion of data collection in the life sciences.

\section{SUPPLEMENTARY MATERIAL}

Supplementary material is linked to the online version of the paper at http://www.nature.com/gim

\section{ACKNOWLEDGMENTS}

We thank Tim Spector and Victoria Vazquez at the BGP for their help with the survey. We are grateful to the German Federal Ministry of Education and Research for supporting this project (grant 01GP1311). We thank two anonymous reviewers for their helpful comments on the manuscript.

\section{DISCLOSURE}

B.P. is an employee of King's College London, which also hosts the BGP. She has no formal connection to the BGP and does not benefit from it financially. The other authors declare no conflict of interest.

\section{REFERENCES}

1. Lozupone CA, Stombaugh JI, Gordon JI, Jansson JK, Knight R. Diversity, stability and resilience of the human gut microbiota. Nature 2012;489:220-230.

2. Human Microbiome Project Consortium. Structure, function and diversity of the healthy human microbiome. Nature 2012; 486: 207-14.

3. Claesson MJ, Jeffery IB, Conde S, et al. Gut microbiota composition correlates with diet and health in the elderly. Nature 2012;488:178-184.

4. Human Microbiome Project Consortium. A framework for human microbiome research. Nature 2012; 486: 215-21.

5. McDonald D, Birmingham A, Knight R. Context and the human microbiome. Microbiome 2015;3:52

6. Del Savio L, Prainsack B, Buyx A. Crowdsourcing the Human Gut. Is crowdsourcing also citizen science? J Sci Com 2016;15:A03.

7. Wooley J, McGowan ML, Teare HJA, et al. Citizen science or scientific citizenship? Disentangling the uses of public engagement rhetoric in national research initiatives. BMC Med Ethics 2016;17:33

8. Su Y, Howard HC, Borry P. Users' motivations to purchase direct-to-consumer genome-wide testing: an exploratory study of personal stories. J Community Genet 2011;2:135-146.

9. Goldsmith L, Jackson L, O'Connor A, Skirton H. Direct-to-consumer genomic testing: systematic review of the literature on user perspectives. Eur J Hum Genet 2012;20:811-816.

10. Vayena E, Gourna E, Streuli J, Hafen E, Prainsack B. Experiences of early users of direct-to-consumer genomics in Switzerland: an exploratory study. Public Health Genomics 2012;15:352-362.

11. Prainsack B, Buyx A. Solidarity in Biomedicine and Beyond. Chapter 4. Cambridge University Press: Cambridge, UK, 2017.

12. Stunkel L, Grady C. More than the money: a review of the literature examining healthy volunteer motivations. Contemp Clin Trials 2011;32:342-352.

13. Adams $M$, McKevitt $C$. Configuring the patient as clinical research subject in the UK national health service. Anthropol Med 2015;22:138-148.

14. McCann SK, Campbell MK, Entwistle VA. Reasons for participating in randomised controlled trials: conditional altruism and considerations for self. Trials 2010;11:31.

15. Prainsack B, Buyx A. A solidarity-based approach to the governance of research biobanks. Med Law Rev 2013;21:71-91.

16. Ipsos MORI. The One Way Mirror: Public Attitudes to Commercial Access to Health Data. Wellcome Trust: London, 2015.

17. Prainsack B, Buyx A. Governing health databases. Solidarity in Biomedicine and Beyond. Cambridge University Press: Cambridge, UK, 2017.

18. Heeney C, Hawkins N, de Vries J, Boddington P, Kaye J. Assessing the privacy risks of data sharing in genomics. Public Health Genomics 2011;14:17-25.

19. Aicardi C, Damjanovicova M, Del Savio L, et al. Could DTC genome testing exacerbate research inequities? Hastings Bioethics Forum. http://www. thehastingscenter.org/response-to-call-for-essays-could-dtc-genome-testingexacerbate-research-inequities/. Accessed 30 June 2016.

20. Galea S, Tracy M. Participation rates in epidemiologic studies. Ann Epidemiol 2007:17:643-653. 